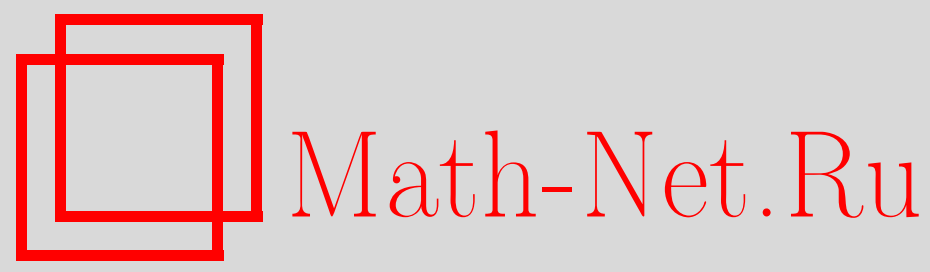

А. А. Махнев, Об одном классе графов без 3-лап, Матем. заметки, 1998, том 63, выпуск 3, 407-413

DOI: https://doi.org/10.4213/mzm1296

Использование Общероссийского математического портала Math-Net.Ru подразумевает, что вы прочитали и согласны с пользовательским соглашением http://www . mathnet.ru/rus/agreement

Параметры загрузки:

IP: 35.173.219.149

26 апреля 2023 г., 14:16:42

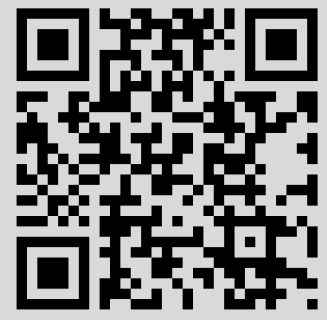




\section{ОБ ОДНОМ КЛАССЕ ГРАФОВ БЕЗ З-ЛАП}

\section{А. А. Махнев}

М. Нуматой описаны реберно регулярные граффы без 3-лап. В таких графах все $\mu$-подграфы являются регулярными одинаковой валентности. В данной работе доказано, что связный графф без 3-лап, в котором все $\mu$-подграфы являются регулярными графами валентности $\alpha>0$, является треугольным графом, графом Шлефли или графом икосаэдра.

Библиографория: 5 названий.

Введение. Мы рассматриваем неориентированные графы без петель и кратных ребер. Для вершины $a$ графа $\Gamma$ через $[a]$ обозначим подграф, индуцированньй $Г$ на множестве всех смежных с $а$ вершин. Соответствующий подграф назьвается окрестностью вериины $а$. Пусть $a^{\perp}-$ шар радиуса 1 с центром $a$. Для подграфа $A$ графа $\Gamma$ через $A^{\perp}$ обозначим $\bigcap_{a \in A} a^{\perp}$.

Через $k_{a}$ обозначим валентность вершины $a$, т.е. число вершин в $[a]$. Граф Г назьвается регулярным валентности $k$, если $k_{a}=k$ для любой вершины $a$ из Г. Для ребра $a c$ графа $\Gamma$ через $\lambda_{a c}$ обозначим число вершин в графе $[a] \cap[c]$. Граф $Г$ назьвается реберно регулярным с параметрами $(v, k, \lambda)$, если $\Gamma$-регулярньй граф валентности $k$ на $v$ вершинах, в котором каждое ребро лежит в $\lambda$ треугольниках $\left(\lambda_{a c}=\lambda\right.$ для любого ребра $a c)$. Подграф, индуцированньй на $[a] \cap[b]$, назовем $\mu$-подграфом, если вершины $a, b$ находятся на расстоянии 2 , а через $\mu(a, b)$ обозначим число вершин в этом подграфе.

Д. Хигмэн предложил программу классификации графов по свойствам их 4-вершинных подграфов [1]. Там же рассмотрены связи между количеством полных подграфов на четырех вершинах (4-клик) и количеством 4-клик с одним удаленным ребром. Мы рассмотрим другой аспект этой программы.

Через $(m, n)$ обозначим полньй двудольный граф с долями порядка $m$ и $n$. Граф $(m, 1)$ назьвается $m$-лапой, если $m \geqslant 2$. При изучении Д. Зейделем сильно регулярных графов, имеющих собственное значение -2 , наибольшие сложности вызвал случай, когда граф не содержал 3-лап [2]. М. Нуматой [3] получена классификация реберно регулярных графов, не содержащих 3-лап. В работе В.В. Кабанова и автора [4] найдены все кореберно регулярные графы без 3-лап. В данной работе получено описание графов без 3-лап, в которых $\mu$-подграфы регулярны валентности $\alpha$. Вершину $a \in \Gamma$ назовем редуиированной, если $\left\{x \in \Gamma \mid a^{\perp} \subseteq x^{\perp}\right\}$ состоит из единственной вершины $a$. Граб $\Gamma$ назьвается редуцированным, если все его вершины редуцированы.

Работа выполнена при финансовой поддержке Российского фонда фундаментальных исследований, грант № 96-01-00488. 
ТЕОРемА. Если Г - связный редуцированный граф без 3-лап, содержащий 3-коклику, в котором $\mu$-подграфы регулярны валентности $\alpha>0$, то Г является одним из следующих графов:

(1) треугольный граф $T(m), m \geqslant 6$;

(2) граф Шлефли (сильно регулярный граф с параметрами $(27,16,10,8)$, дополнительный к точечному графу обобщенного четырехугольника $G Q(2,4))$;

(3) граф икосаәдра.

СлЕдСТВИЕ. Пусть Г - реберно регулярный граф без 3-лап. Тогда граф Г является либо реберным графом регулярного графа без треугольников ( и н-подграфы коклики из не более чем двух вершин), либо сильно регулярным графом без 3-коклик, либо графом из заключения теоремы.

Граф на множестве пар $X \times Y$ назьвается $(m \times n)$-графом, если $|X|=m,|Y|=n$, а пары $\left(x_{1}, y_{1}\right)$ и $\left(x_{2}, y_{2}\right)$ смежны тогда и только тогда, когда $x_{1}=x_{2}$ или $y_{1}=y_{2}$. Иногда $(m \times n)$-графф называется прямоугольной решеткой. При $m=n$ граф называется решетчатым. Треугольным графом $T(m)$ назьвается граф с множеством неупорядоченных пар из $X$ в качестве вершин; $|X|=m$ и пары $\{a, b\},\{c, d\}$ смежны только, если они имеют общий элемент. Реберно регулярньй граф с параметрами $(v, k, \lambda)$, в котором каждая пара вершин $a, b \mathrm{c} d(a, b)=2$ имеет $\mu$ обших смежных с ними вершин, т.е. $|[a] \cap[b]|=\mu$, называется вполне регулярным графом с параметрами $(v, k, \lambda, \mu)$. Вполне регулярный граф диаметра 2 называется сильно регулярным. Далее подграф из Г будет означать индуцированный подграф.

Графом Тәйлора называется вполне регулярный граф диаметра 3 , в котором каждая вершина лежит в $a^{\perp} \cup b^{\perp}$ для любых двух вершин с $d(a, b)=3$. Граф икосаэдра - это граф Тэйлора, в котором окрестность любой вершины является пятиугольником. Граф Тервиллигера - это граф, в котором $\mu$-подграфы являются кликами одного порядка.

1. Вспомогательные результаты. В этом пункте $\Gamma$ - связньй графф без 3-лап.

Лемма 1.1. Пусть $а \in \Gamma$. Тогда верны следующие утверэсдения:

(1) если $b, c$-не смехсные вериины из $[a]$, то $[a] \subseteq b^{\perp} \cup c^{\perp}$;

(2) если $b, c-$ не смежные вериины из $\Gamma-a^{\perp}$, то $\mu$-подграф $[b] \cap[c]$ не пересекaem $[a]$.

ДокАЗАтЕЛьство. Если вершина $x \in[a]$ не лежит в $b^{\perp} \cup c^{\perp}$, то граф $\{a ; b, c, x\}$ является 3 -лапой и (1) доказано.

Пусть вьполнено условие (2) леммы. Если $x$ - вершина из $[a] \cap[b] \cap[c]$, то граф $\{x ; a, b, c\}$ является 3 -лапой.

ЛЕМма 1.2. Если асbd - четырехугольник графа Г, то выполняются следующие утверждения:

(1) $a^{\perp} \cup b^{\perp}=c^{\perp} \cup d^{\perp}$;

(2) если $\mu$-подграфы $[a] \cap[b],[c] \cap[d]$ являются регулярными графами одинаковой валентности, то $k_{a}+k_{b}=k_{c}+k_{d}, \mu(a, b)=\mu(c, d)$.

ДокАЗАтЕЛьство. По лемме $1.1[a],[b]$ содержатся в $c^{\perp} \cup d^{\perp}$. Утверждение (1) следует теперь из симметричности четырехугольника. 
Пусть $\alpha$ - валентность указанных $\mu$-графов. По лемме $1.1 k_{a}=2+\lambda_{a c}+\lambda_{a d}-\alpha$, $k_{b}=2+\lambda_{b c}+\lambda_{b d}-\alpha$. Выписав аналогичные выражения для $k_{c}, k_{d}$, получим первое равенство в (2).

Число вершин в графе $a^{\perp} \cup b^{\perp}$ равно $2+k_{a}+k_{b}-\mu(a, b)$. Это же число согласно (1) равно $2+k_{c}+k_{d}-\mu(c, d)$. Отсюда следует второе равенство в $(2)$.

ЛЕмма 1.3. Если асbd - четырехугольник графа $\Gamma$ и граф $[b] \cap[e]$ содержит несмежние вериины для $е \notin a^{\perp} \cup b^{\perp}$, то $x^{\perp}=b^{\perp}$ для любой не смежной $с а$ вериины $x \in[c] \cap[d]$.

ДокАЗАТЕЛЬство. По лемме $1.2 a^{\perp} \cup b^{\perp}=a^{\perp} \cup x^{\perp}$; в частности, $[x]$ содержит $[b] \cap[e]$. Снова по лемме $1.2 e^{\perp} \cup b^{\perp}=e^{\perp} \cup x^{\perp}$. Таким образом, $b^{\perp}$ и $x^{\perp}$ совпадают вне $a^{\perp} \cap e^{\perp}$. Утверждение леммы следует теперь из леммы 1.1 (2).

Лемма 1.3 доказана В.В. Кабановым. Им же предложено следующее утверждение, которое может оказаться полезным и для некоторых графов с 3-лапами. Скажем, что граф $\Sigma$ обладает четырехугольным свойством, если для любого четырехугольника $a c b d$ графа $\Sigma$ графы $a^{\perp} \cup b^{\perp}$ и $c^{\perp} \cup d^{\perp}$ совпадают.

ЛЕмма 1.4. Пусть $\Sigma$ - граф, обладающий четырехугольным свойством, и $a, b, e$ - 3-коклика, не лежащая в окрестности никакой вериины. Если пары $a, b$ и $b, e$ лежат в четырехугольниках, причем $c, d$ - не смежные вериины из $[a] \cap[b]$, то $b^{\perp} \subseteq x^{\perp}$ для любой не смежсной с а вериины $x \in[c] \cap[d]$.

ДокАЗАТЕЛЬСТВО является простой модификацией рассуждений из доказательства леммы 1.3.

Следующее утверждение справедливо для произвольных графов Тервиллигера.

Лемма 1.5. Пусть $\Sigma$ - связный редуцированный граф Тервиллигера, $а$ - вериина из $\Sigma$. Тогда для любого ребра bс из [a] найдется вериина $d$, принадлежащая $a^{\perp}$ и несмежнная $с$ в и

ДокАЗАтЕЛЬство. Пусть $a^{\perp}$ содержится в $b^{\perp} \cup c^{\perp}$. По редуцированности графа разность $[b]-c^{\perp}$ содержит вершину $x$ из $[a],[c]-b^{\perp}$ содержит $y$ из $[a]$. Заметим, что вершины $x$ и $y$ не смежны, иначе $[x] \cap[c]$ содержит несмежные вершины $b$ и $y$.

Теперь граф $[x] \cap[y]$ содержится в $[b] \cap[c]$. В самом деле, если $z$ - несмежная с $b$ вершина из $[x] \cap[y]$, то $z$ смежна с $c$ по предположению и $[b] \cap[z]$ содержит несмежные вершины $x$ и $c$. Теперь $[y] \cap[b]$ содержит весь $\mu$-граф $[x] \cap[y]$ и вершину $c$. Противоречие. Лемма доказана.

ЛЕмма 1.6. Пусть Г - связный редуцированный граф Тервиллигера без 3-лап с $\mu>1$. Тогда $Г-$ граф икосаэдра.

ДоКАЗАТЕЛЬСТво. Если $a, b \in \Gamma$ и $d(a, b)=2$, то для любой вершины $c \in[a] \cap[b]$ получим равенство $k_{c}=2+\lambda_{a c}+\lambda_{b c}-(\mu-1)$.

Для вершины $c \in \Gamma$ положим $\Lambda_{c}=[c]$ и покажем, что $\Lambda_{c}$ - регулярньй граф валентности $\lambda$. Из условия $\mu>1$ и редуцированности $Г$ следует, что граф $\Lambda_{c}-$ связньй графф Тервиллигера. Пусть $x y$ - ребро графа $\Lambda_{c}$. По лемме $1.5 \Lambda_{c}$ содержит вершину $z$, не смежную с $x, y$. Как и вьше, $k_{c}=3-\mu+\lambda_{c x}+\lambda_{c z}$, поэтому $\lambda_{c x}=\lambda_{c y}$ и $\Lambda_{c}-$ регулярньй граф валентности $\lambda$. 
По связности графа $Г$ получим, что $\lambda_{u w}=\lambda$ для любого ребра $u w$. Регулярность графа Г следует теперь из равенства, приведенного в первом абзаце доказательства.

По теореме Тэйлора-Ливингстона $[5,1.5 .3]$ вполне регулярньй неполньй графф Тервиллигера без 3-лап либо имеет $\mu=1$, либо является графом икосаэдра.

ЛЕмма 1.7. Если $\Delta$ - связный граф, в котором все $\mu$-графы регулярны одинаковой валентности, не являются кликами и имеют одинаковое число вершин, то $\Delta$ является регулярным графом.

ДокАЗАТЕЛьство. Пусть $a, b$ - пара смежных вершин из $\Gamma, c$ - произвольная вершина из $[a]-[b]$. Если $\mu=|[b] \cap[c]|$ и $\alpha$ - валентность $\mu$-графа $[b] \cap[c]$, то вершина $c$ смежна с $\mu-\alpha-1$ вершиной из $[b]-a^{\perp}$. Теперь число ребер из $[b]-a^{\perp}$ в $[a]-b^{\perp}$ равно $\left|[b]-a^{\perp}\right|(\mu-\alpha-1)$, а число ребер из $[a]-b^{\perp}$ в $[b]-a^{\perp}$ равно $\left|[a]-b^{\perp}\right|(\mu-\alpha-1)$. Отсюда $|[b]-[a]|=|[a]-[b]|$ и, следовательно, $|[a]|=|[b]|$. Лемма доказана.

2. Четырехугольники в графах без 3-лап. Пусть до конца работы граф Г является контрпримером к теореме. Скажем, что пара вершин $a, b$ из Г является сильной, если граф $[a] \cap[b]$ не является кликой. Вершину $a$ назовем сильной, если она образует сильную пару с некоторой вершиной $b$.

ЛЕмма 2.1. Граф Г содержит четырехугольники.

ДоКАЗАТЕльСТво. Если Г не содержит четырехугольников, то Г является графом Тервиллигера с $\mu=\alpha+1$. По лемме $1.6 \Gamma$-граф икосаэдра. Лемма доказана.

Зафиксируем четырехугольник $a c b d$ графф $Г$ и положим $\Delta=\{a, b, c, d\}, \Lambda=a^{\perp} \cup b^{\perp}$.

ЛЕмма 2.2. Если асbе - геодезический 3-nуть из $\Gamma$, то $\mu$-подграфы $[a] \cap[b],[c] \cap[e]$ являются кликами.

ДокАЗАтЕльство. Допустим, что $[a] \cap[b]$ содержит несмежные вершины $c, d$. По лемме $1.2 e \in c^{\perp} \cup d^{\perp}$. Противоречие с тем, что $e \in \Gamma_{3}(a)$.

ЛЕМма 2.3. Г не содержит несвязных $\mu$-подграфов.

ДоКАЗАТЕЛЬСТво. Пусть граф $[a] \cap[b]$ несвязен. Тогда он состоит из двух изолированных клик $C, D$ порядка $\alpha+1$. Пусть $c, e-$ две вершины из $C$. Согласно лемме 1.2 графы $c^{\perp}, e^{\perp}$ совпадают вне $d^{\perp}$ для любой вершины $d \in D$. Выберем вершину $x \in[c]-e^{\perp}$. Без ограничения общности, $x \in[a] \cap D^{\perp}$. Заметим, что $[x] \cap[b]$ содержит изолированную клику $D$, поэтому $[x] \cap[b] \cap c^{\perp}$ лежит в $e^{\perp}$. Но тогда в графе $[e] \cap[x]$ вершина $c$ смежна еще с $a$, и валентность $c$ в этом графе не меньше $\alpha+1$. Значит, $c^{\perp}=e^{\perp}$, что противоречит редуцированности графа.

Лемма 2.4. Пусть вериина $g \in \Gamma_{2}(a)-\Lambda$. Тогда граф $[a] \cap[g]$ пересекает $[c]$ $u[d]$.

ДокАЗАтЕЛЬСтво. Допустим, что $[g] \cap[a] \subseteq[c]$. Для вершины $w \in[a] \cap[g]$ получим включение $[w] \cap a^{\perp} \subseteq c^{\perp}$, иначе $[w]$ содержит 3-коклику: $c, g$ и вершину из $[a]-c^{\perp}$. Если граф $[a]$ содержит пару несмежных вершин $x, y$ из $[d] \cap[w]$, то $x, y \in[c] \cap[d]$. По лемме $1.1[w] \subseteq x^{\perp} \cup y^{\perp}$ и $g \in \Lambda$, противоречие.

Значит, $[d] \cap[w]$ содержит $(\alpha+1)$-клику из $a^{\perp}$, лежащую в $[c] \cap[d]$, и граф $[c] \cap[d]$ не связен. Противоречие с леммой 2.3 . 
Лемма 2.5. Пусть вериина $g \in \Gamma_{2}(a)-\Lambda$. Тогда

(1) $[g] \cap[x] \neq[g] \cap[y]$ для любого ребра ху графа $\{a, b\} \cup([a] \cap[b])$;

(2) граф $\{a, b\} \cup([a] \cap[b])$ является полным многодольным с долями порядка 2 ;

(3) nара $g, x$ является сильной для $x \in\{a, b\} \cup([a] \cap[b])$.

ДокАЗАТЕльСТво. Если $x \in\{a, b\}$, то утверждение $(1)$ следует из леммы 2.4. Пусть $x, y \in[a] \cap[b]$ и $[g] \cap[x]=[g] \cap[y]$. Для вершины $w \in[x] \cap[g]$ получим равенство $[w] \cap[x]=[w] \cap[y]$. Отсюда $[g] \cap[x]$ является $(\alpha+1)$-кликой и $x^{\perp}, y^{\perp}$ совпадают вне $[a] \cap[b]$. Пусть $z$ - несмежная с $x$ вершина из $[a] \cap[b] \cap[y]$.

Выберем $w \in[g] \cap[x] \cap[a]$. Тогда вершина $y$ имеет валентность $\alpha$ в графе $[z] \cap[w]$, причем эти $\alpha$ вершин лежат в $[x]$. Противоречие с тем, что вершина $y$ смежна еше с вершиной $b$ из $[x] \cap[z]$. Утверждение (1) доказано.

Если вершина $x \in\{a, b\} \cup([a] \cap[b])$ не смежна с двумя вершинами $y, z \in\{a, b\} \cup([a] \cap[b])$, то согласно лемме $1.2[g] \cap[z]=[g] \cap[y]$, что противоречит утверждению (1) леммы. Это доказьвает утверждение (2).

Пусть граф $[a] \cap[g]$ является кликой. Без ограничения общности, можно считать, что $|[g] \cap[a] \cap[c]| \geqslant(\alpha+1) / 2$. Выберем вершину $w \in[g] \cap[a] \cap[d]$. Очевидно, $[w]$ содержит $\alpha / 2+1$ вершин из $c^{\perp} \cap d^{\perp}$, поэтому валентность вершины $a$ в графе $[w] \cap[c]$ не меньше $(2 \alpha+1) / 2$. Полученное противоречие доказывает, что $a, g$ - сильная пара. Ввиду утверждений $(1),(2)$ леммы любую вершину $x \in\{a, b\} \cup([a] \cap[b])$ можно взять в роли $a$; это доказьвает (3).

ЛЕмма 2.6. Для любой сильной вершины $x$ әраф $\Gamma_{3}(x)$ nуст.

ДокАЗАтЕЛьство. Достаточно показать, что граф $\Gamma_{3}(a)$ пуст. Пусть $e \in \Gamma_{3}(a)$ и afge-некоторьй 3-путь. Если $g \in[b]$, то весь $\mu$-подграф $[a] \cap[g]$ лежит в $[b]$, иначе $[g]$ содержит 3-коклику. В этом случае граф $[a] \cap[g]$ является связной компонентой графа $[a] \cap[b]$, что противоречит лемме 2.3 .

Значит $g \notin \Lambda$ и по лемме $2.5(3)$ пара $a, g$ является сильной. Противоречие с леммой 2.2.

Лемма 2.7. Пусть $\Gamma \neq \Lambda$. Тогда

(1) любая вершина из $[a]-[b]$ смежсна с некоторой вершиной в $\Gamma-\Lambda$;

(2) вериина $x \in \Gamma-a^{\perp}$ образует сильную пару с а, причем $\Gamma \neq a^{\perp} \cup x^{\perp}$.

ДокАЗАтЕЛЬСтво. Пусть $e \in \Gamma-a^{\perp}, y \in[a]-[b]$ и $[y] \subseteq \Lambda$. Ввиду леммы 2.5 (2) $[y]$ содержит не менее $\alpha / 2+1$ вершин из $[e] \cap[a]$. По лемме $1.1[y] \cap[e]$ не пересекает $[b]$, поэтому $[y] \cap[e] \subseteq[a]$. Но если $u, w-$ не смежные вершины из $[y] \cap[e]$, то по лемме $2.5 u^{\perp} \cup w^{\perp}=a^{\perp} \cup e^{\perp}$. Противоречие с тем, что $y \notin[e]$. Значит, $[y] \cap[e]$ является $(\alpha+1)$-кликой из $[a] \cap[e]$. Противоречие с леммой 2.3. Утверждение $(1)$ доказано.

Пусть $x \in \Gamma-a^{\perp}$ и граф $[a] \cap[x]$ является кликой. Ввиду леммы $2.5 x \in \Lambda$. Если $x$ смежна с некоторой вершиной $e \in \Gamma-\Lambda$, то $[b] \cap[e]$ содержит не смежную с $x$ вершину $z$. По лемме $2.5(3)$, примененной к 4-угольнику $b x e z$, получим, что $a, x$ - сильная пара. Значит, $[x]$ не содержит вершин из $\Gamma-\Lambda$. Противоречие с утверждением (1) леммы.

ЛЕмма 2.8. $\Gamma=a^{\perp} \cup b^{\perp} \partial л я$ любой сильной пары $a, b$. 
ДокаЗАтЕЛЬСтво. Допустим, что $\Lambda \neq \Gamma$. Пусть $x \in[a]$. Если $x \in[a] \cap[b]$, то $x$ - сильная вершина и $x^{\perp} \cup y^{\perp}=\Lambda$ для не смежной с $x$ вершины $y \in[a] \cap[b]$. Если же $x \in[a]-[b]$, то по лемме $2.7 x, b$-сильная пара и $\Gamma \neq x^{\perp} \cup b^{\perp}$. По лемме 2.7 , примененной к вершине $x$ вместо $a, x, z$ - сильная пара для любой вершины $z \in \Gamma-x^{\perp}$. По связности графа любой $\mu$-подграф из Г является полньм многодольным графом с долями порядка 2 на $\alpha+2$ вершинах. По лемме 1.7 граф Г регулярен. По теореме Кабанова и автора [4] $\Gamma$ - прямоугольная решетка, треугольный граф или граф Шлефли. Но в решетке $\alpha=0$. Противоречие с выбором $Г$. Лемма доказана.

3. Окончание доказательства. Пусть $v$ - число вершин в графе Г. Ввиду леммы $2.8 v=2+k_{a}+k_{b}-\mu(a, b)$ для любой сильной пары $a, b$.

Лемма 3.1. Пусть $a, b$ - сильная пара $u[a] \cap[b]$ содержит вериину $x$, лежащую в 3-коклике $x, y, z$. Тогда $\{a, b\} \cup([a] \cap[b])-$ полный многодольный граф с долями порядка 2.

ДоКАЗАТЕЛЬСТво. Пусть вьполнены условия леммы. Без ограничения общности, $y \in[a]-[b], z \in[b]-[a]$. Выберем не смежную с $x$ вершину $w$ из $[a] \cap[b]$. Пусть $[w]$ содержит $\beta+1$ вершин из $[x] \cap[y], \gamma+1$ вершин из $[x] \cap[z]$. В графе $[a] \cap[b]$ вершина $w$ имеет валентность $\alpha$; поэтому $[a] \cap[b] \cap[w]$ содержит $\alpha-\beta-\gamma$ вершин из $[y] \cap[z]$.

В графе $[x] \cap[w]$ вершина $a$ имеет валентность $\alpha$, причем $\beta$ из этих вершин лежат в $[y]$, а остальные $\alpha-\beta$ вершин лежат в $[z]$. Симметрично, $[x] \cap[w] \cap[b]$ содержит $\gamma$ вершин из $[z]$ и $\alpha-\gamma$ вершин из $[y]$. Таким образом, $[x]$ пересекает $[a] \cap[b]$ по $\alpha-\beta$ вершинам из $[z]$ и по $\alpha-\gamma$ вершинам из $[y]$. Следовательно, $\alpha=\beta+\gamma$.

Теперь $\mu(w, x)=2+\beta+\gamma=2+\alpha$. Поэтому $\{x, w\} \cup([x] \cap[w])=\{a, b\} \cup([a] \cap[b])-$ полный многодольный графф с долями порядка 2 и $\beta=\gamma=\alpha / 2$. Лемма доказана.

ЛЕмма 3.2. Если а, $b$ - сильная пара, то $[a] \cap[b]$ не пересекает 3-коклик.

ДокАЗАТЕЛЬСтво. Пусть выполнены условия леммы 3.1. Без ограничения обшности, $y \in[a]-[b], z \in[b]-[a]$. Выберем не смежную с $x$ вершину $w$ из $[a] \cap[b]$.

Граф $[a] \cap[z]$ лежит в $x^{\perp} \cup y^{\perp}$. Из доказательства леммы 3.1 следует, что $[a] \cap[z]$ содержит $\alpha / 2$ вершин из $[x] \cap[z]$ и $1+\alpha / 2$ вершин из $[y] \cap[z]$. Отсюда граф $[a] \cap[z]$ является кликой; поэтому $[a],[c]$ совпадают на $[y] \cap[z]$ для $c \in[a] \cap[x] \cap[z]$. Как и вьше, $[y] \cap[c]$ содержит $\alpha / 2$ вершин из $[x] \cap[z]$ и $1+\alpha / 2$ вершин из $[x] \cap[y]$. Снова граф $[y] \cap[c]$ является кликой. Но если $[c]$ содержит смежную с $b$ вершину $d \in[x] \cap[y]$, то $d$ смежна с единственной вершиной $w$ из $[c] \cap[y] \cap[z]$. Таким образом, $\alpha=2$.

Пусть $c \in[a] \cap[x] \cap[z], u$ - отличная от $w$ смежная с $c$ вершина из $[y] \cap[z]$. Тогда граф $[b] \cap[u]$ не является кликой (вершина $d \in[y] \cap[z]-\{u, w\}$ не смежна с $c$ ) и содержит не смежную с $z$ вершину. Но $[b] \cap[u]$ лежит в $\left(x^{\perp} \cup y^{\perp}\right) \cap\left(x^{\perp} \cup z^{\perp}\right)$; поэтому $[b] \cap[u]$ содержит вершину из $[x] \cap[y]$. Противоречие $c$ тем, что $b$ смежна с единственной вершиной из $[x] \cap[y]$, которая не смежна с $u$. Лемма доказана.

Завершим доказательство теоремы. Пусть $a c b d$ - четырехугольник, $x, y, z-3$-коклика. Одна из вершин $a, b$ смежна с единственной вершиной из этой 3 -коклики. Пусть для определенности $a$ не смежна с $y, z$. Тогда $a, y, z$ является 3 -кокликой, причем $a \in[c] \cap[d]$ для сильной пары $c, d$. Противоречие с леммой 3.2. Теорема доказана.

Докажем следствие. Пусть $\Gamma$ - реберно регулярный граф без 3-лап с параметрами $(v, k, \lambda)$. Согласно лемме $1.1 \mu$-подграфы из $Г$ регулярны валентности $2+2 \lambda-k$. Если $2+2 \lambda-k=0$, то окрестность любой вершины состоит из двух изолированных 


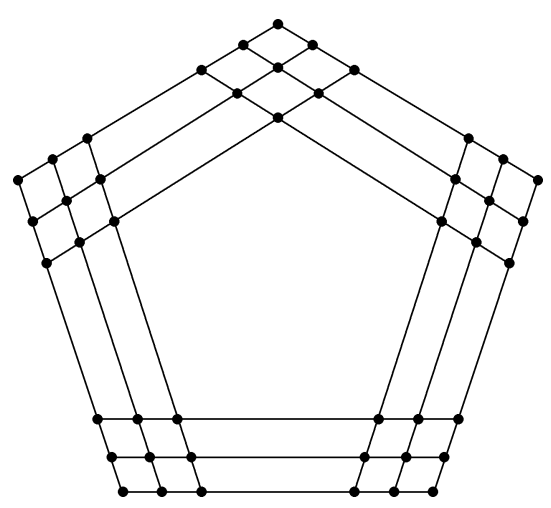

Рис. 1. Граф $P(3)$

$(\lambda+1)$-клик. В этом случае граф $Г$ является реберньм графом регулярного графа без треугольников. Если же $2+2 \lambda-k>0$, то либо Г является сильно регулярным графом без 3-коклик, либо граф Г удовлетворяет условиям теоремы. Отметим в заключение, что реберно регулярньй граф без 3-лап диаметра 2 является либо сильно регулярным графом, либо графом $P(m)$ (см. граф $P(3)$ на рис. 1) [3].

\section{СПИСОК ЦИТИРОВАННОЙ ЛИТЕРАТУРЫ}

[1] Hestenes M.D., Higman D.G. Rank 3 groups and strongly regular graphs // Proc. SIAM-AMS. 1971. V. 4. P. 141-159.

[2] Seidel J. J. Strongly regular graphs with $(-1,1,0)$ adjacency matrix having eigenvalue 3 // Linear Algebra Appl. 1968. V. 1. P. 281-298.

[3] Numata M. On a characterization of a class of regular graphs // Osaka J. Math. 1974. V. 11. P. 389-400.

[4] Кабанов В. В., Махнев А. А. Кореберно регулярные графы без 3-лап // Матем. заметки. 1996. T. 60. № 4. C. 495-503.

[5] Brouwer A. E., Cohen A. M., Neumaier A. Distance-Regular Graphs. Berlin: Springer, 1989.

Институт математики и механики УрО РАН, г. Екатеринбург

Поступило

E-mail: mak@top.imm.intec.ru

18.04 .96 